Association for Information Systems AIS Electronic Library (AISeL)

Wirtschaftsinformatik Proceedings 2003

Wirtschaftsinformatik

September 2003

\title{
Mobile Adaptation with Multiple Representation Approach as Educational Pedagogy
}

Kinshuk

MasseyUniversity, New Zealand, kinshuk@inspire.net.nz

Tiong-Thye Goh

Victoria University, New Zealand

Follow this and additional works at: http://aisel.aisnet.org/wi2003

\section{Recommended Citation}

Kinshuk and Goh, Tiong-Thye, "Mobile Adaptation with Multiple Representation Approach as Educational Pedagogy" (2003). Wirtschaftsinformatik Proceedings 2003. 39.

http://aisel.aisnet.org/wi2003/39 
In: Uhr, Wolfgang, Esswein, Werner \& Schoop, Eric (Hg.) 2003. Wirtschaftsinformatik 2003: Medien - Märkte - Mobilität, 2 Bde. Heidelberg: Physica-Verlag

ISBN: 3-7908-0111-9 (Band 1)

ISBN: 3-7908-0116-X (Band 2)

(C) Physica-Verlag Heidelberg 2003 


\title{
Mobile Adaptation with Multiple Representation Approach as Educational Pedagogy
}

\author{
Kinshuk \\ Massey University, New Zealand \\ Tiong-Thye Goh \\ Victoria University, New Zealand
}

\begin{abstract}
This paper illustrates the concept of Multiple Representation (MR) approach and its application in mobile adaptation. The paper first reviews several techniques for web page level content adaptation. Most of these techniques have been used in existing applications but they are very generic and lack the guidelines for deployment of educational pedagogy needed in learning environments. The Multiple Representation approach fills this gap by providing guidelines for content adaptation in e-learning and mobile learning environments. We further formulate, extend and apply MR approach in a prototype of a mobile quiz module to illustrate the synergy between mobile adaptation and MR approach.
\end{abstract}

Keywords: Mobile adaptation, educational Pedagogy, handheld devices

\section{Introduction}

The success of the learning process in an educational system depends on many factors such as how the system presents the domain knowledge to the learner, how it changes its presentation in terms of complexity and granularity according to learner's progress, and quite importantly, how it takes into account the user device and user preference profiles. Tutoring strategies are the major source of taking decisions regarding domain knowledge presentation. A set of effective and efficient tutoring strategies leads to the creation of educational framework. Recognising the device and preference profiles allows adaptation engine to make decision about users choices. A good adaptation engine will seamlessly adapt to user environment, anytime and anywhere.

The need for suitable educational framework in the use of multimedia technology in educational systems has been emphasised by many researchers. Educational 
software is expected to be not only a teaching and learning resource, but also a carrier of the instructional strategies $\left[\mathrm{Ada}^{+} 96, \mathrm{p} .182\right]$. Therefore, the design of such system and its presentation should consider learning theories and concepts, the pedagogies that apply to those concepts, and how they impact instruction design and practice. In additional, mobile learning adaptation should also be part of the design consideration to increase learning opportunity. Without mobile adaptation, these systems will have limited scope to offer in the new wave of mobile learning. The content adaptation on mobile learning has not received much attention till now, despite many advances in the use of multimedia technologies in such devices.

Multimedia technology can contribute to the success of learning only if it can adequately represent the tasks and concepts of the domain knowledge. The Multiple Representation (MR) approach, presented in this paper, is predominantly dependent on the framework of tutoring strategies in which it is being applied. The next section discusses the general concept of content adaptation and reviews some practical approaches to provide the foundation for the linkage between MR and content adaptation.

\section{General Framework for Content Adaptation}

Content adaptation can be approached in two different directions as illustrated in figure 1 (a) and (b).

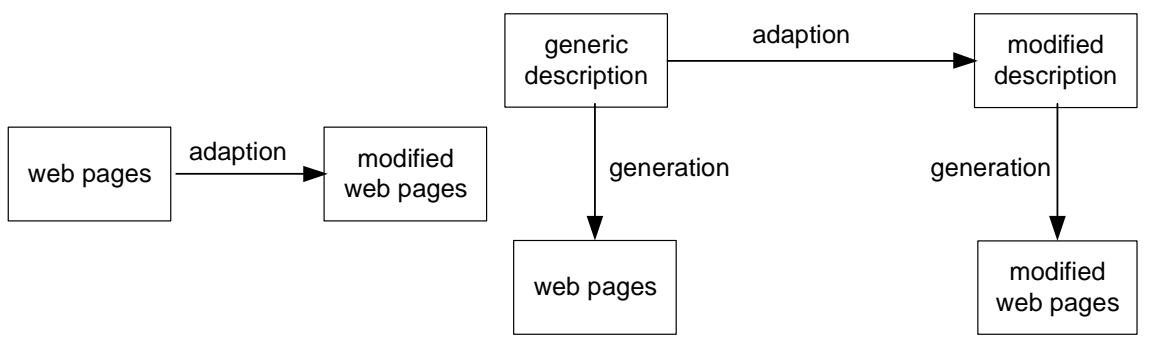

(a)

(b)

Figure 1: Flow of content adaptation

The first class of approaches deal with the problem on the level of pages, i.e. we assume that the web-based service is fully implemented by web pages that can be displayed by a standard browser on a standard computer display. Then adaptation has to take HTML as the format of its input. In the UWA framework [Fin ${ }^{+} 02$, p.431; Kap ${ }^{+}$2, p.79; Schw02] the adaptation can be expressed by event-conditionaction rules represented in a customisation framework. 
The second class of approaches handle the problem on a conceptual level, i.e. we assume to start with a generic abstract description of the service's content, from which the actual web pages could be generated. Adaptation now takes the abstract description as its input and derives another abstract description. The pages generated out of this modified specification are better suitable for specific clients, in particular mobile clients.

A lot has been written about content adaptation on the level of pages. The most prominent approaches are re-authoring [BiSc97] and transcoding [Bha ${ }^{+} 98 ;$ p.118; $\mathrm{Smi}^{+}$99]. Reauthoring applies functions to web-page descriptions, which will result in new descriptions that are better suited for various kinds of clients. Transcoding aims at direct manipulation of the HTTP stream. Based on these techniques several commercial products and system prototypes have been implemented such as Digestor [BiSc97], Spyglass [Spyg01], Intel QuickWeb [Inte98], Mobiware [Ang ${ }^{+}$98, p.32], TranSend [Fox ${ }^{+} 98 \mathrm{a}$, p.10], WingMan [Fox ${ }^{+}$98b], and Power Browser [Buy ${ }^{+}$99].

Adaptivity on the level of page descriptions can be achieved more easily if data content, navigation links and presentation are separated from each other, e.g., by using XML, XLink and XSL. The work in [Che $\left.{ }^{+} 01\right]$ presents an interesting approach to detect objects in pages and their categories. Adaptation rules are then applied to these objects. We will review these page-based techniques for content adaptation in more detail in the next section.

\section{Page-Level Content Adaptation Techniques}

Among the various published techniques for content adaptation, re-authoring, transcoding and the functional-based object model (FOM) are the most relevant representatives. We will briefly review these techniques.

\subsection{Re-authoring}

The idea of re-authoring is to apply functions to web-page descriptions, which will result in new descriptions that are better suited for various kinds of clients. As web-pages usually present a mix-up of content, navigation and presentation, it is desirable to first split pages into these components. In a second step adaptation functions can be applied to the isolated components. These functions may be designed particularly for mobile clients. The third step consists of re-integrating the components into a new web-page description.

On the basis of HTML pages it is very difficult, if not impossible to provide algorithms for the first step. Therefore, it is advantageous, if page descriptions exist in the form of XML documents. These provide the description of content, 
whereas navigation and presentation are separately described by XLink and XSL respectively.

The content adaptation functions could change or remove elements in the XML document. These techniques are known as transformation and elision [BiSc97]. The follow-on navigation adaptation functions would reduce the links to those needed for the new content. Finally, applying XSL would result in a new presentation.

The obvious disadvantage of the re-authoring technique is that it is nongeneric. Even if XML is used, transformation and elision functions have to written separately for each site and each new end-device. Furthermore, if parts of the page description have been removed due to the restrictions imposed by the client, the user may nevertheless be interested to obtain the omitted information in follow-on pages. So far re-authoring does not provide any assistance for this.

\subsection{Transcoding}

Transcoding aims at direct manipulation of the HTTP stream. Modifying the HTTP streams and changing its content in situ is called active transcoding and is done dynamically without user intervention. Transcoding can be done on both upstream and downstream HTTP strings. A known implementation of this technique is the MOWSER (Mobile Browser) system [Bha ${ }^{+} 98$, p.118], an Apache proxy server agent written in Perl, in which the proxy modifies the in-coming HTTP stream.

For the modification itself transcoding rules are used, which are kept separate from the HTML pages. These rules take into account the user preferences $\left[\mathrm{Bha}^{+} 98, \mathrm{p} .118\right]$ and annotations $\left[\mathrm{Hor}^{+} 00\right]$.

In the MOWSER system user preferences and client capabilities are stored on the server. The modification and update of preferences is done via a CGI form at the web site maintained by the proxy. The proxy then fetches files with the most suitable format for the mobile client. The system assumes that different formats are available for content adaptation. This is not an issue, as different formats can be created on the fly and cached in the server for future requests. Transcoding of images and video is done using scaling, sub-sampling or sub-key frames. The transcoding of HTML pages is done by eliminating unsupported tags according to the stored user preferences. However, a severe limitation of the MOWSER system is that it does not affect navigation.

Annotation is a way of providing hints for a transcoding engine to make better decisions on the content adaptation [Hor $\left.{ }^{+} 00\right]$. Basically, this method uses some predefined descriptors to define the transcoding rules. Descriptors should be stored separately from the HTML files. Annotation plays the role of a mediating representation, which provides semantics to be shared between meta-content 
authors and a content adaptation engine. A potential advantage of an annotationbased transcoding approach is the possibility of content adaptation based on semantics that cannot be achieved by approaches based on web-page syntax.

The principles discussed for re-authoring also apply to the annotation-based transcoding, i.e., decomposition (isolation), partial replacement of content (distillation or elision), and combination (re-mapping). The implementation reported in $\left[\mathrm{Hor}^{+} 00\right]$ uses the RDF framework for implementing the annotation descriptors and Xpath / Xpointer for associating an external description with a portion of an existing document. Thus, benefits resulting from using XML-related technologies have already been realised. The annotation uses a predefined vocabulary comprising alternatives, splitting hints and selection criteria.

Annotation-based transcoding has proven to be useful for mobile systems, which are task-oriented such as mobile assessment, mobile quiz or mobile forms. The problem with annotation-based transcoding, however, is that it is applicationspecific, whereas customisation using a mark-up language is very limited. It is also difficult to generalise the approach, so it will most likely not be suitable for interactivity and navigation logic.

\subsection{The Functional-Based Object Model}

The FOM approach aims at detecting objects in pages and their categories before applying adaptation rules to them. This approach is an attempt to describe the intentions behind pages and to shift content adaptation to a semantic level. Nevertheless, the approach remains on the level of page descriptions rather than switching to a conceptual abstraction.

The rationale is that every object in a web site serves for certain functions, which are either basic or specific functions. According to this FOM distinguishes between objects, which themselves can be basic or composite, and object categories. Based on this, a complete web-site is transformed first into a FOM model, and adaptation rules are applied to the model rather than the web-pages.

A basic object is the smallest element in a hypermedia. It is determined by its presentation, semantics, decoration, hyperlink associated with it, and its interaction. Basic object can be grouped into finite sets, which are considered as composite objects. A composite object $\mathrm{C}=\left\{\mathrm{O}_{1}, \ldots, \mathrm{O}_{\mathrm{N}}\right\}$ is further described by clustering and presentation relationships.

The specific function of an object in a given application environment is represented by its category, which directly reflect the authors' intention. Examples of such object categories in FOM are information objects, navigation objects, decoration objects, special function objects, and page objects. 
The FOM method requires basic object detection to be performed first, then to generate the necessary category objects. Composite objects are detected by layout analysis of the web pages using an image pattern detection algorithm. Then, content adaptation rules are applied to the objects producing the adapted pages. The FOM method uses different rules for each object category.

\subsection{Summary}

The numerous approaches of content adaptation provide hints to a general class of web page adaptation. But these techniques do not provide adequate guidelines for multimedia representation in the domains of e-learning or m-learning. We argue that the Multiple Representation (MR) approach can be adopted to improve and enrich user experience in an educational setting.

\section{The Multiple Representation Approach}

Multiple Representation (MR) approach tackles the presentation of domain content in three ways: multimedia objects selection; navigational objects selection; and integration of multimedia objects.

\subsection{Multimedia Objects Selection}

The MR approach facilitates the presentation of domain content to the learner with suitable multimedia objects, as and when required, according to learner's preferences, current level of domain competence, and familiarity with the learning environment. Various recommendations are described below.

\subsubsection{Task Specificity and Learner's Competence}

MR approach recommends the selection of multimedia objects on the basis of the tasks to be carried out. For example, audio is good to stimulate imagination, video clips for action information, text to convey details, and diagrams for conveying ideas [Alty91, p.31]. The selection of objects should also consider the level of learner's domain competence. For example, an abstract concept could initially be introduced with the help of an animation, and later on, at higher level of abstraction, the representations could be more complex requiring more cognitive processing [RoSc97]. 


\subsubsection{Expectations}

The selection of multimedia objects should take care of the expectations of the learner and the domain about representation of the tasks. For example, a learner who is looking for an overview of middle ear structure may expect to see just a graphic representation, but from domain's point of view, textual details are also necessary to emphasise some intricate details. The system in that case should try to present graphical representation along with textual intricate details.

\subsubsection{Reference \& Revisits of Already Learnt Domain Content}

In the process of learning it is many times necessary and desirable to refer already learnt domain content in different contexts. The MR approach favours revisiting the same domain content in different contexts, using similar multimedia objects as used before, for following reasons.

1. Referencing enforces links between concepts (the one currently being learnt and the referred one);

2. It enhances the mental model of previously learnt concept and helps in generalising its applicability in multiple situated scenarios;

3. It provides ease in learning current concept by making familiarisation with past learning experiences.

\subsubsection{Use of Multi-Sensory Channels}

The selection of objects should adequately use the visual, aural and tactile senses of the learner. If any of the sensory channels is not being used at the time of learning, the chances of getting distraction due to this channel are high.

\subsubsection{Context Based Selection of Multimedia Objects}

When there are more than one multimedia objects available for representation of the same task or concept, the presentation should use the most suitable object in that particular context.

\subsubsection{Authenticity of Multimedia Objects}

Schematic diagrams and animations of the processes may not show the real objects but they are helpful in understanding the underlying processes. In such cases, the system should keep the learner aware about the authenticity of the representation by suitable messages. 


\subsection{Navigational Objects Selection}

The navigation in typical educational systems takes place through various links provided in the system. [Rada95, p.1] pointed out that the link does not say what happens to the screen when the user activates the link. The important point to consider is the proper match of the learner's expectations of outcome while activating a link with the presentation of actual resulting interface.

According to the MR approach, the selection of links should not deviate learner's attention from the main task of learning. The existence of link should be as transparent as possible. The MR approach identifies six types of navigational links concerning learning processes.

1. Direct successor link leads to the successive domain unit in knowledge hierarchy within current context.

2. Parallel concept link leads to the analogous domain unit for comparative learning or to the unit related to another aspect of currently being learnt domain content.

3. Fine grained unit link leads to very contextual fine details of the domain content once some missing or mis-conceptions are identified in learners understanding [PaKi97, p.161].

4. Glossary link leads to a pop-up "spring loaded" module [Nie196] in exploration process, which is available only till learner is interested in it and is explicitly doing something to keep it active (such as pressing the mouse button).

5. Excursion link leads to a learning unit outside the current context [Kas+97, p.114].

6. Problem link leads to the problems related to current conceptual unit.

Different types of links should be clearly identified for their types [Ben ${ }^{+} 97$, p.197] and their representations should be consistent throughout the system.

\subsection{Integration of Multimedia Objects}

In many situations, the presentation of the domain content demands more than one multimedia object at a time on the screen for suitable representation. A number of studies have also showed the improvement in learning through more than one multimedia object for the same domain content compared to single object. ADGA Group [Adga96, p.1] suggested that learning improves as the number of complementary stimuli and different cognitive resources used to present learning content increases. But not all possible combinations of multimedia objects are adequate from learning point of view. Consideration should be on how best to combine multiple multimedia objects in relation to different learning tasks 
[RoSc97]. Following are some recommendations regarding integration of multimedia objects to be observed during design process of educational systems.

- There should not be more than one observation multimedia object at a time on the screen, except for comparison studies, where more than one active observation is required.

- The integration of multimedia objects should be complimentary to each other and should be synchronised [RoSc97]. Care should also be taken not to present the same material with more than one multimedia object.

- Integration of decision intensive objects, e.g. flowcharts, with any other multimedia object is not recommended due to possible high cognitive loading.

- Integration of dynamic observation objects (such as animations) with static observation objects (such as text) should be such that the learner should not be forced to observe both of them by the same sensory channel at the same time.

\section{MR Extension}

The MR approach provides a good set of recommendations in the design of multimedia learning environment from the perspective of adapting user's preferences and current domain knowledge. However it does not particularly focus on mobile adaptation. Hence the MR approach is further extended to include mobile consideration to provide a more unifying approach, as described below.

1. Mobile device capability selection

Consideration needs to be given in the MR to respond to different device capability of the end users. For example low end PDAs have no capability of displaying image and video but high end PDAs can do both. MR selection needs to be adjusted to accommodate the whole spectrum of devices.

2. Mobile user preference selection

While high end PDAs can display rich multimedia content, user preference sometime required that option to be turned off. The MR approach needs to consider alternatives as per the mobile user preferences.

3. Mobile learner profile extraction and conversion

MR approach needs to consider Mobile Learning profile to be extracted from the PC based learner profile and converted to suit mobile environment. It can not be assumed that MR selection in one environment will be automatically suited to another environment. 
4. Content adaptation and selection

MR approach in content adaptation is further complicated by the possiblity of synchronisation and content pre-fectching, in additional to the real time on-line adaptation. Hence MR approach needs to consider these content adaptation scenarios. For example, if certain multimedia objects are pre-fetched and are available in the device's cache, MR approach should consider using those objects while recommending content selection.

\section{A Case Study - Mobile Learning}

Mobile learning is defined as the ability to learn anytime and anywhere. In our context, it is defined as the ability of using handheld devices to access learning information. Mobile learning has received a lot of attention these days as the next wave of learning. Several research issues regarding the pedagogical effects of wireless instructional devices have been investigated [ $\mathrm{Liu}^{+} 02$, p.63; MiHo02, p.117; Luc $^{+} 02$, p.107; Wayc02, p.177; Mifs02, p.112; Gay $^{+} 01$, p.257). Yet, educational pedagogy has not been the central theme in these research efforts. With the extension of MR approach, we use a simple m-learning quiz case study to illustrate some of the consideration of MR approach.

The case study proposed here is an architecture and prototype design based on XML/XSLT technologies. The system performs adaptation based on user devices and user learning profiles with considerations of MR approach.

\subsection{Server Side Implementation Techniques}

There are two main approaches to implement MR approach for device adaptation in mobile applications: single pipeline approach and multiple pipeline approach. The single pipeline approach requires the server page to generate device specific language for the browser (figure 2). The server page performs device identification and channels the XML data for the appropriate style sheets. Several style sheets are needed to perform the transformation. 


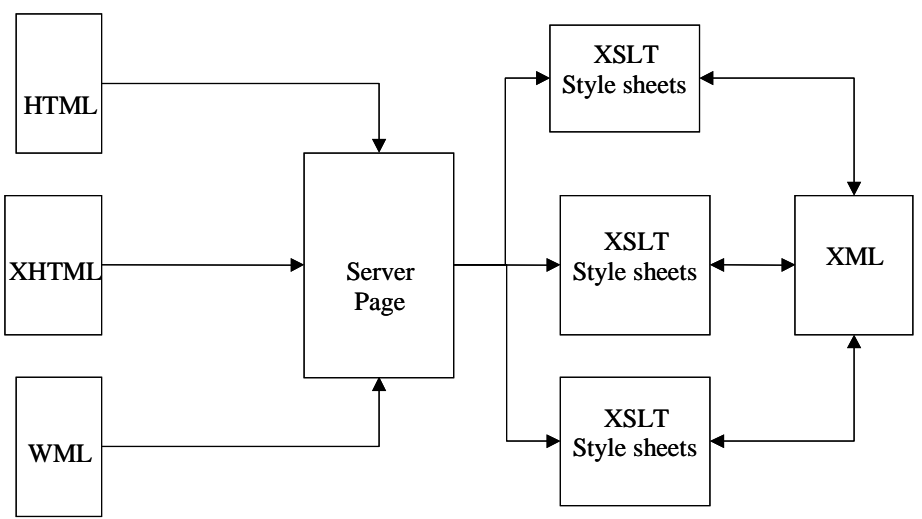

Figure 2: A single pipeline approach

The multiple pipelines approach uses a set of client-specific sever pages to generate the device specific output (figure 3). Several sever pages are needed to perform the transformation. There is a development cost in creating and maintaining server-side objects that represent the application's data abstractions, which is not required in the single pipeline approach.

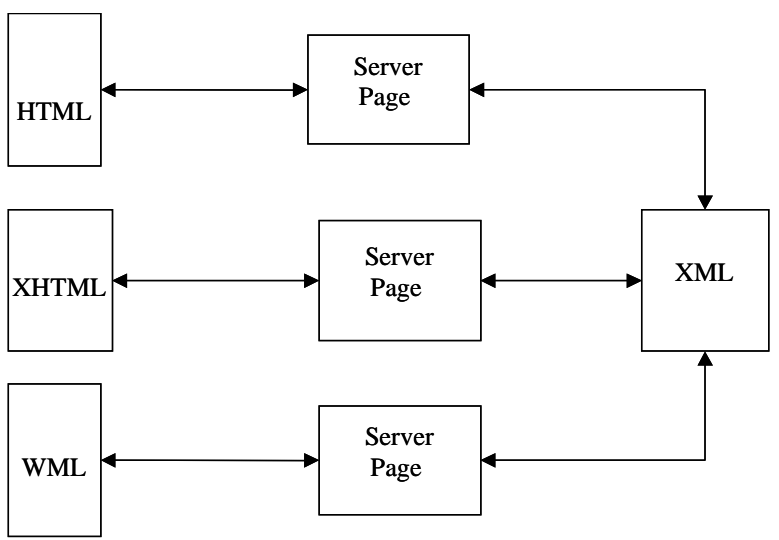

Figure 3: Multiple pipeline approach

\subsection{Methodology}

The MR approach used in this implementation is illustrated in Figure 4 with MR extension for mobile devices. Several technological solutions have been available to implement the prototype of dynamic and adaptive test system. Sever side techniques include Java Servlets, JSP, ASP, PHP and other proprietary authoring 
tool such as macromedia flash with action script. Other enhanced severs such as CoCoon or Xalan are also capable of implementing device adaptable system. However many of these tools are costly, require a steep learning curve, and the deployment process is slow.

MR Approach

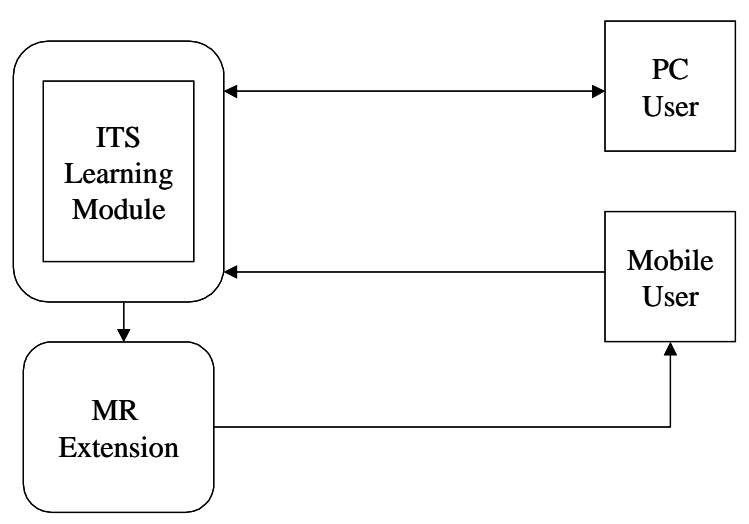

Figure 4: MR approach extension to mobile learning

Our design is based on following guidelines. First, it must be easy to develop. Second, it must have potential for reuse. Third, it must minimize learning curve. Fourth, it can be deployment rapidly. Lastly, no XSLT support should be needed at the client so as to be compatible with a broad variety of devices. Our design uses ASP with IIS 5 server. The design first identifies the potential mobile user devices. These are desktop PCs, PDAs (iPAQ), and mobile phones with Openwave browser. Other mobile device types can be easily added later. Device identification uses a simple http header string identification technique included in the ASP page. Subsequently, we develop the quiz content template using XML and its DTD. Multiple multimedia objects such as text, image, and video at different levels of complexity are placed in the revision folder to match mobile user with MR extension consideration. Next we develop the style sheets suitable for each device types. We use MSXML as our transform engine for parsing XML documents. To provide an adaptive system, the users profile is separately stored in an XML file and the style sheet function is used to match the required level of difficulty from the user profile. The current implementation is to adapt to the level of difficulty according to the user profile and render the content which includes multimedia objects according to the user device. Figure 5 shows an overview of the system. 


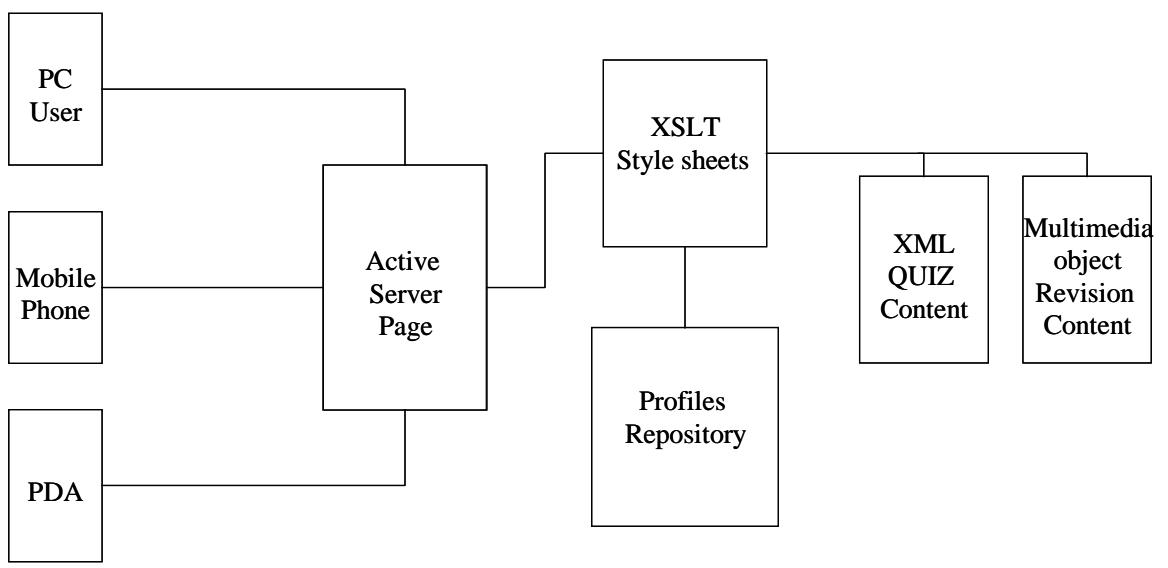

Figure 5: Mobile learning module architecture

Figure 6 show a display of mobile quiz access via mobile phone. Since the device is not capable of displaying rich multimedia, the revision page produces text based information about the topic. MR extension for mobile learning allows this adaptation to be done seamlessly.

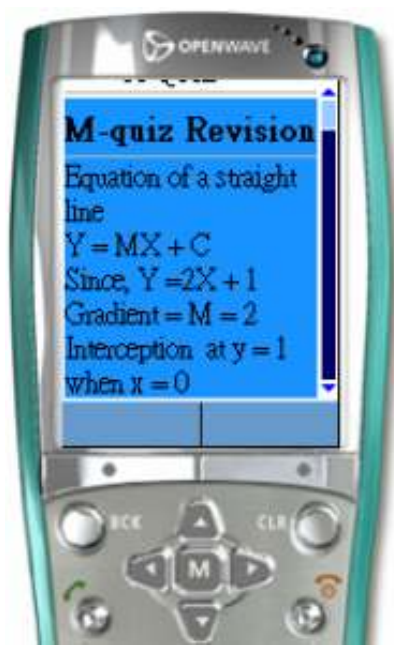

Figure 6: Revision page with mobile phone adaptation

Figure 7 shows the same display of mobile quiz accessed via PDA. Since PDA is capable of displaying multimedia-rich information, the revision page produces video-based information according to MR extension. 


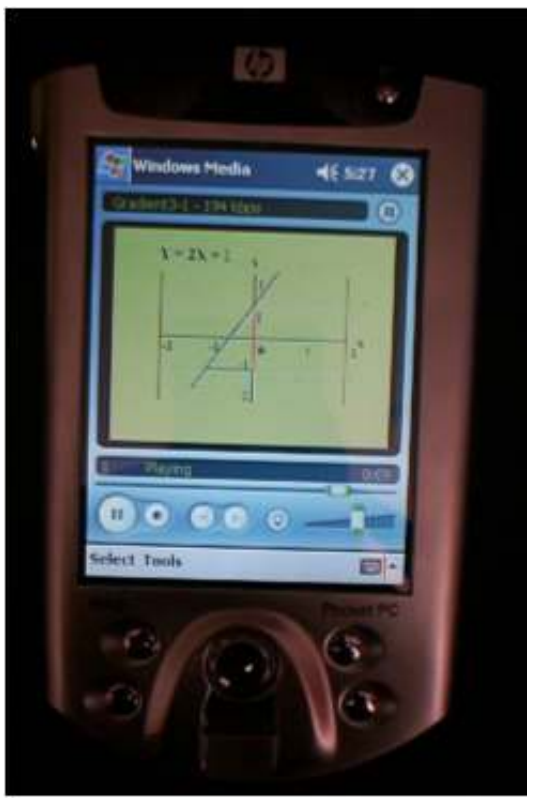

Figure 7: Revision page with PDA adaptation

\section{Conclusion}

This paper described the concept of Multiple Representation approach in applying content adaptation techniques using educational pedagogy. The approach fills the gap created by the lack of educational pedagogy in the general web content adaptation in the learning systems. With the advent of $3 \mathrm{G}$ and $4 \mathrm{G}$ network, the use of mobile device will be increasing exponentially, with particular expansion in the learning process where learner can learn anytime and anywhere. The MR approach is extended to provide m-learning consideration with extension towards the device selection, user preference selection, learner profile selection and content selection. The prototype m-learning quiz case study illustrates the benefits of the MR approach. The aim at this stage is to verify the validity of the conceptual approach. Further research will verify the generality of the MR extensions to variety of devices and user requirements. 


\section{References}

[Ada ${ }^{+}$96] Adams, E. S., Carswell, L., Ellis, A., Hall, P., Kumar, A., Meyer, J., Motil, J.: Interactive multimedia pedagogies: Report of the working group on interactive multimedia pedagogy. SigCUE Outlook, 24 (1, 2 \& 3), 1995: pp. 182-191.

[Adga96] ADGA Group: Instruction and design principles for multimedia computer based training, Technical Report, 1996: Ontario: ADGA.

[Alty91] Alty, J. L.: Multimedia - What is it and how do we exploit it? In D. Diaper, N. Hammond (Eds.), People and Computers IV, Cambridge: CUP, 1991, pp. 31-46.

[Ang ${ }^{+}$98] Angin, O., Campbell, A. T., Kounavis, M. E., Liao, R. R.-F.: The Mobiware Toolkit: Programmable support for adaptive mobile networking. IEEE Personal Communications, 5 (4), 1998: pp. 32-43.

[Ben $\left.{ }^{+} 97\right]$ Benyon, D., Stone, D., Woodroffe, M.: Experience with developing multimedia courseware for the World Wide Web: the need for better tools and clear pedagogy. International Journal of Human-Computer Studies, 47, 1997: pp. 197-218.

[Bha $\left.{ }^{+} 98\right]$ Bharadvaj, H., Joshi, A., Auephanwiriyakul, S.: An Active Transcoding Proxy to Support Mobile Web Access. 17th IEEE Symposium on Reliable Distributed Systems, October 1998, pp. 118-123.

[BiSc97] Bickmore, T., Schilit, B.: Digestor: Device Independent Access to the World Wide Web. http://www.fxpal.com/papers/bic97/, 1997, Download 2003-03-01.

$\left[\mathrm{Buy}^{+} 99\right]$ Buyukkokten, O., Molina, H. G., Paepcke, A., Winograd, T.: Power Browser: Efficient web browsing for PDAs. Stanford University Digital Libraries Lab. http://www-db.stanford.edu/ orkut/papers/pb1.pdf, 1999, Download 2003-03-01.

[Che $\left.{ }^{+} 01\right]$ Chen, J., Zhou, B., Shi, J., Zhang, H., Wu, Q. (2001). Functional-based Object Model Towards Website Adapation. WWW10 Conference, May 1-5, 2001, Hong Kong. http://www10.org/cdrom/papers/296/, 2001, Download 2003-03-01.

[Fin ${ }^{+}$02] Finkelstein, A., Savigni, A., Kappel, G., Retschitzegger, W., Kimmerstorfer, E., Schwinger, W., Hofer, T., Proll, B., Feichtner C.: Ubiquitous web application development - a framework for understanding. 6th World Multiconference on Systemics, Cybernetics and Informatics (SCI 2002). Orlando (Florida, USA), 2002, pp. 431-438.

[Fox ${ }^{+}$98a] Fox, A., Gribble, S. D., Chawathe, Y., Brewer, E. A.: Adapting to network and client variation using active proxies: lessons and perspectives. IEEE Personal Communication, 5 (4), 1998a: pp. 10-19.

[Fox ${ }^{+}$98b] Fox, A., Goldberg, I., Gribble, S. D., Lee, D. C., Polito, A., Brewer, E. A.: Experience with top gun wingman: A proxy-based graphical web browser for the user palmpilot. Proc. IFIP International Conference on Distributed Systems Platforms and Open Distributed Processing, http://www.cs.utah.edu/ retrac/cs7460/wingman.pdf, 1998b, Download 2003-03-01.

$\left[\mathrm{Gay}^{+} 01\right]$ Gay, G., Grace-Martin, M, Hembrooke H.: The effects of wireless computing in collaborative learning. International Journal of Human-Computer Interaction, 13 (2), 2001: pp. 257-276. 
[Hor $\left.{ }^{+} 00\right]$ Hori, M., Kondoh, G., Ono, K., Hirose, S., Singhal, S.: Annotation-Based Web Content Transcoding. IBM, http://www9.org/w9cdrom/169/169.html, 2000, Download 2003-03-01.

[Inte98] Intel QuickWeb: http://www.intel.com/pressroom/archive/releases/IN011998.htm, 1998, Download 2003-03-01.

[Kap ${ }^{+}$2] Kappel G, Retschitzegger W, Kimmerstorfer E., Proll B., Schwinger W., and Hofer T.: Towards a generic customisation model for ubiquitous web applications. Proc. 2nd Int. Workshop on Web Oriented Software Technology, Malaga, 2002, pp. 79104.

[Kas ${ }^{+}$97] Kashihara, A., Kinshuk, Oppermann, R., Rashev, R., Simm, H.: An Exploration Space Control as Intelligent Assistance in Enabling Systems. In Z. Halim, T. Ottmann, Z. Razak (Eds.), International Conference on Computers in Education Proceedings, VA: AACE, 1997, pp. 114-121.

[Liu $\left.{ }^{+} 02\right]$ Liu, T. C., Wang, H.-Y., Liang, J. K., Chan, T. W., Yang, J. C. (2002). Applying Wireless Technologies to Build a Highly Interactive Learning Environment. Proc. of the IEEE International Workshop on Wireless and Mobile Technologies in Education, Los Alamitos, IEEE Cs Press, 2002, pp. 63-70.

[Luc $\left.{ }^{+} 02\right]$ Luchini, K., Curtis, M., Quintana, C., Soloway, E.: Supporting Learning in Context: Extending Learner-Centered Design to the Development of Handheld Educational Software. Proc. of the IEEE International Workshop on Wireless and Mobile Technologies in Education, Los Alamitos, IEEE Cs Press, 2002, pp. 107-111.

[MiHo02] Milrad, M., Hoppe, U.: C-Notes: Designing a Mobile and Wireless Application to Support Collaborative Knowledge Building. Proc. of the IEEE International Workshop on Wireless and Mobile Technologies in Education, Los Alamitos, IEEE Cs Press, 2002, pp. 117-120.

[Mifs02] Mifsud, L.: Alternative learning arenas - pedagogical challenges to mobile learning technology in education. Proc. of the IEEE International Workshop on Wireless and Mobile Technologies in Education, Los Alamitos, IEEE Cs Press, 2002, pp. 112-116.

[Nie196] Nielsen J.: Features missing in current web browsers. http://www.sun.com/ 950701/columns/alertbox/newfeatures.html, 1996, Download 2003-03-01.

[PaKi97] Patel, A., Kinshuk: Granular Interface Design : Decomposing Learning Tasks and Enhancing Tutoring Interaction. In M. J. Smith, G. Salvendy R. J. Koubek (EDs.), Advances in Human Factors/Ergonomics - 21B - Design of Computing Systems: Social and Ergonomic Considerations. Amsterdam: Elsevier Science B. V., 1997, pp. 161-164.

[Rada95] Rada, R.: Hypertext, multimedia and hypermedia. In P. Baird (Ed.), The New Review of Hypermedia and Multimedia. London: Taylor Graham Publishing, 1995, pp. $1-21$

[RoSc97] Rogers, Y., Scaife, M.: How can interactive multimedia facilitate learning? http://www.cogs.susx.ac.uk/users/yvonner/ecoihome/IMMI.html, 1997, Download 2003-03-01. 
[Schw02] Schwinger, W.: The UWA approach to modeling ubiquitous web applications. IST mobile and wireless telecommunications summit, http://newton.ee.auth.gr/ summit2002/papers/SessionW5/2602141.pdf, 2002, Download 2003-03-01.

[Smi $\left.{ }^{+} 99\right]$ Smith, J., Mohan, R., Li, C.: Scalable multimedia delivery for pervasive computing. ACM Multimedia, http://www.ee.columbia.edu/ jrsmith/html/pubs/ acmmm99.pdf, 1999, Download 2003-03-01.

[Spyg01] Spyglass: http://www.opentv.com/support/primer/prism.htm, 2001, Download 2003-03-01.

[Wayc02] Waycott, J (2002). An Evaluation of the Use of PDAs for Reading Course Materials. Proc. of the IEEE International Workshop on Wireless and Mobile Technologies in Education, Los Alamitos, IEEE Cs Press, 2002, pp. 177-178. 